\title{
Using Latent Class Regression in the Evaluation of Awareness Level of Esfahanian Women 20 to 65 Years on Different Components of Breast Cancer
}

\author{
Ghasem Yadegarfar ${ }^{1}$, Hamid Iranshahi ${ }^{1,}$ \\ ${ }^{1}$ School of Health, Isfahan University of Medical Sciences, Isfahan, Iran \\ * Corresponding author: Hamid Iranshahi, School of Health, Isfahan University of \\ Medical Sciences, Isfahan, Iran.E-mail: hamidiranshahi@yahoo.com
}

DOI: $10.21859 /$ mci-supp-76

\section{Keywords:}

Breast Cancer

Awareness

Risk Factor

Early Symptoms Screening

Latent Class Regression

\begin{abstract}
Introduction: In Iran, the age of breast cancer is 10 years lower than in western countries and patients often refer to diagnostic and therapeutic centres in advanced stages of the disease which is associated with a low level of awareness of signs and screening methods among women. Various epidemiological studies have shown that correct awareness of the different dimensions of the disease leads to a change in the quality of life and increased survival of the individuals in that community. The purpose of this paper is to present accurate education needs in various women $>$ groups and by providing effective strategies to increase awareness of the different dimensions of the disease, preventing breast cancer, or if it occurs, is discovered and treated at an early stage and ultimately reduced the burden of the disease in community.

Materials and Methods: This study, using data collected in 2012, was conducted as a cross-sectional study on 10,000 Esfahanian women aged 20 to 65 years. Of these, 9260 women were eligible for the study and the data from the questionnaire were analysed using Latent Class Regression.

Results: The mean age of the subjects in this study was $37.5 \pm 11.7$ years. In evaluating the factors affecting the identification of risk factors for breast cancer, age variable had very little effect on the placement of subjects at different levels. The chance for women with positive family history of breast cancer in class I and II is 30\% higher than class III.

Conclusions: The most effect on the awareness levels of all aspects of breast cancer is the place of people residence (urban / rural). So that individuals living in urban areas had a greater chance of being placed at a higher level of aware than rural residents, which due to the unjust distribution of wealth and educational.
\end{abstract}

